\title{
Robust Tensegrity Polygons
}

\author{
János Geleji · Tibor Jordán
}

Received: 12 October 2012 / Revised: 26 July 2013 / Accepted: 19 August 2013 /

Published online: 31 August 2013

(C) Springer Science+Business Media New York 2013

\begin{abstract}
A tensegrity polygon is a planar cable-strut tensegrity framework in which the cables form a convex polygon containing all vertices. The underlying edge-labeled graph $T=(V ; C, S)$, in which the cable edges form a Hamilton cycle, is an abstract tensegrity polygon. It is said to be robust if every convex realization of $T$ as a tensegrity polygon has an equilibrium stress which is positive on the cables and negative on the struts, or equivalently, if every convex realization of $T$ is infinitesimally rigid. We characterize the robust abstract tensegrity polygons on $n$ vertices with $n-2$ struts, answering a question of Roth and Whiteley (Trans Am Math Soc 265:419-446, 1981) and solving an open problem of Connelly (Recent progress in rigidity theory, 2008).
\end{abstract}

Keywords Tensegrity framework $\cdot$ Tensegrity polygon $\cdot$ Rigid framework

\section{Introduction}

A tensegrity graph $T=(V ; B, C, S)$ is an edge-labeled simple undirected graph with vertex set $V=\left\{v_{1}, v_{2}, \ldots, v_{n}\right\}$ whose edge-set is partitioned (labeled) into pairwise disjoint sets $B, C$, and $S$, referred to as bars, cables, and struts, respectively. Elements of $E=B \cup C \cup S$ are the members of $T$. A two-dimensional tensegrity framework $(T, p)$ is a tensegrity graph $T=(V ; B, C, S)$ together with a map $p: V \rightarrow \mathbb{R}^{2}$. We say that $(T, p)$ is a realization of $T$ in $\mathbb{R}^{2}$. We can also view $(T, p)$ as a geometric graph in the plane in which the lengths of the bars are fixed, cables do not increase in length,

\footnotetext{
J. Geleji · T. Jordán (凶)

Department of Operations Research, Eötvös University, Pázmány Péter sétány 1/C,

Budapest 1117, Hungary

e-mail: jordan@cs.elte.hu

J. Geleji

e-mail: janos@geleji.hu
} 
and struts do not decrease in length. Rigidity properties of tensegrity frameworks are important in mathematics as well as in engineering and have been studied by several researchers. In this paper we shall focus on the infinitesimal rigidity and the existence of nowhere-zero equilibrium stresses in special tensegrity frameworks, motivated by questions posed by Roth and Whiteley in 1981 [10] and Connelly in 2008 [5].

Let $(T, p)$ be a tensegrity framework. A proper equilibrium stress, or proper stress, for short, on $(T, p)$ is an assignment of scalars $\omega_{i j}$ to the members for which $\omega_{i j} \geq 0$ if $v_{i} v_{j} \in C$ and $\omega_{i j} \leq 0$ if $v_{i} v_{j} \in S$ and such that for each $v_{i} \in V$

$$
\sum_{j \mid v_{i} v_{j} \in E} \omega_{i j}\left(p\left(v_{i}\right)-p\left(v_{j}\right)\right)=\mathbf{0} .
$$

We say that the stress is non-trivial if it is non-zero on at least one member. A strict proper stress on $(T, p)$ is a proper stress satisfying $\omega_{i j}>0$ if $v_{i} v_{j} \in C$ and $\omega_{i j}<0$ if $v_{i} v_{j} \in S$. (In some papers stress and proper stress are used instead of proper stress and strict proper stress, respectively.)

Let $q: V(T) \rightarrow \mathbb{R}^{2}$ be a map, assigning infinitesimal velocities to the vertices of $T$. We say that $q$ is an infinitesimal motion of $(T, p)$ if the scalar product $\left(p\left(v_{i}\right)-\right.$ $\left.p\left(v_{j}\right)\right)\left(q\left(v_{i}\right)-q\left(v_{j}\right)\right)$ is zero (resp. non-positive, non-negative) for all bars $v_{i} v_{j} \in B$ (resp. cables $v_{i} v_{j} \in C$, struts $v_{i} v_{j} \in S$ ) of $T$. The framework $(T, p)$ is infinitesmally rigid in $\mathbb{R}^{2}$ if the vector space of the infinitesimal motions is three-dimensional, i.e. it contains the trivial infinitesimal motions only, arising from translations and rotations of the whole framework. The following key result, due to Roth and Whiteley, establishes a connection between proper stresses and infinitesimal rigidity.

Theorem 1.1 [10, Theorem 5.2] Let $(T, p)$ be a tensegrity framework. Then $(T, p)$ is infinitesmally rigid if and only if $(\bar{T}, p)$ is infinitesimally rigid and there is a strict proper stress on $(T, p)$, where $(\bar{T}, p)$ denotes the underlying bar framework obtained from $(T, p)$ by replacing all members by bars.

A tensegrity polygon $(T, p)$ is a tensegrity framework in $\mathbb{R}^{2}$ with the following properties:

(i) the vertices of $(T, p)$ are distinct and form the set of vertices of a strictly convex polygon $P$ in the plane,

(ii) the cables of $(T, p)$ are the edges of $P$,

(iii) the set of bars is empty.

Thus every strut of a tensegrity polygon is a diagonal of $P$, i.e. it runs through the interior of $P$. In the rest of the paper convex will always mean strictly convex.

The existence of a non-trivial or strict proper stress in a tensegrity polygon $(T, p)$ can be used to show different forms of rigidity in tensegrity frameworks and other structures, see Sect. 1.2 for related results.

An abstract tensegrity polygon is a tensegrity graph $T=(V ; C, S)$ with no bars in which the cables form a Hamilton cycle $H$ of the underlying graph. This cycle of cables will be called the boundary cycle of $T$. We shall assume that the numbering of the vertices follows the cyclic ordering induced by $H$. A planar realization of an 
Fig. 1 A tensegrity polygon on four vertices

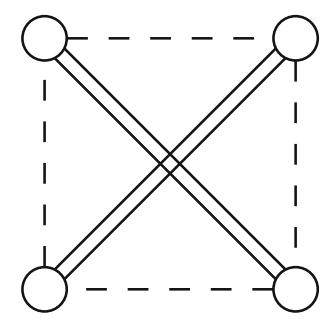

Fig. 2 The Grünbaum polygon on five vertices. In each figure we shall use filled circles to mark those vertices which are incident with at least two struts
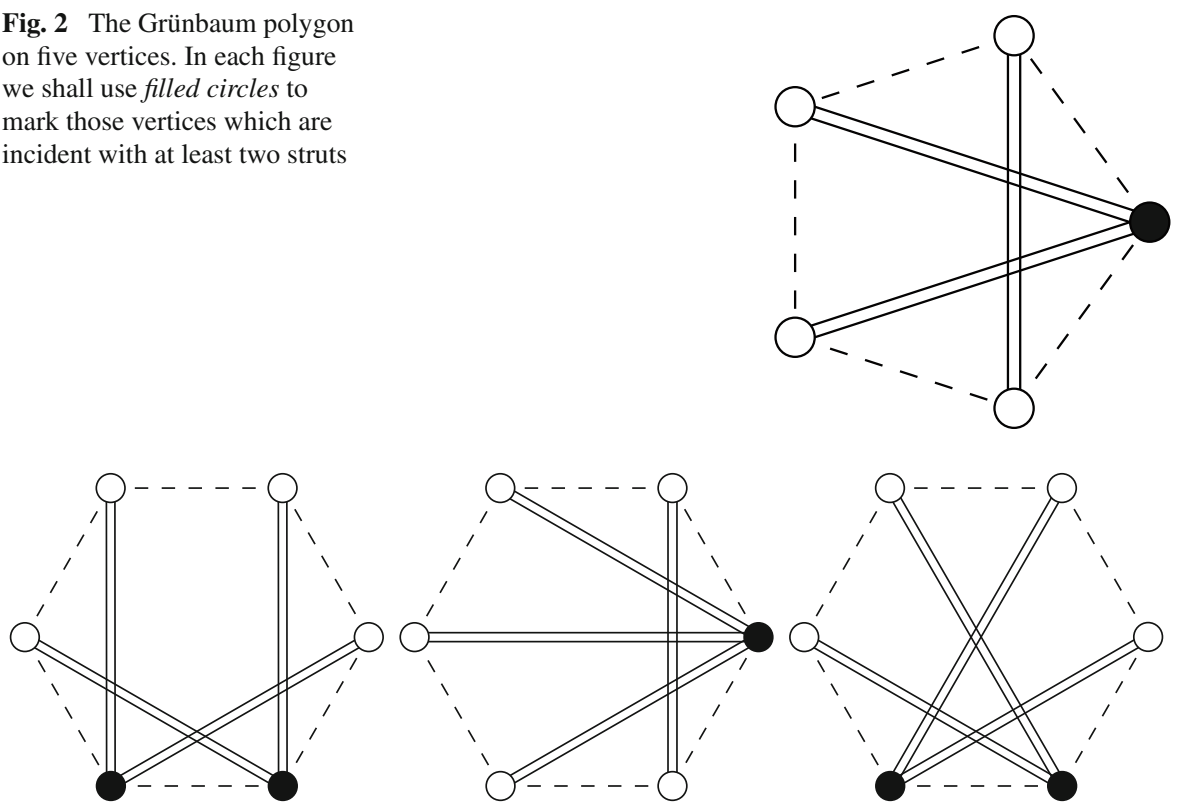

Fig. 3 The Cauchy polygon, the Grünbaum polygon, and a generalized Grünbaum polygon on six vertices. They are all robust and stable

abstract tensegrity polygon $T$ in which the boundary cycle forms a convex polygon will be called a convex realization of $T$. Thus every convex realization of an abstract tensegrity polygon is a tensegrity polygon, and every tensegrity polygon is a convex realization of some abstract tensegrity polygon.

Examples The most well-studied families of (abstract) tensegrity polygons are the Cauchy polygons (in which the struts are of the form $v_{i} v_{i+2}, 1 \leq i \leq n-2$ ) and the Grünbaum polygons (in which the struts are $v_{1} v_{3}$ plus $v_{2} v_{i}, 4 \leq i \leq n$ ). See Fig. 1, which is the smallest Cauchy and Grünbaum polygon at the same time, and also Fig. 2 and the first two polygons in Fig. 3. Roth and Whiteley [10] consider generalized Grünbaum polygons, too, in which two adjacent vertices are chosen along the boundary cycle and every other vertex is joined by a strut to exactly one of these vertices in such a way that each of the chosen vertices becomes incident with at least one strut. Note that in all these examples we have $|V|-2$ struts and hence $2|V|-2$ members in total. 

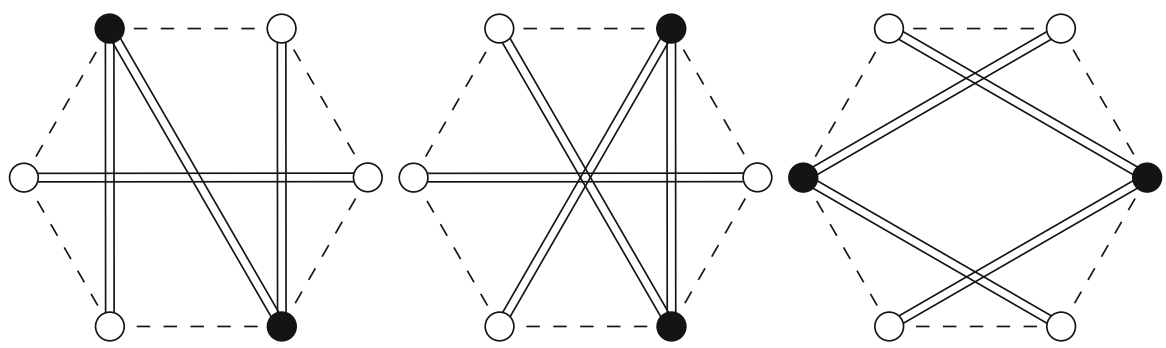

Fig. 4 Non-robust tensegrity polygons on six vertices

We call an abstract tensegrity polygon $T$ strong if every convex realization $(T, p)$ has a non-trivial proper stress. It is said to be robust if every convex realization $(T, p)$ has a strict proper stress. It is stable if every convex realization $(T, p)$ is infinitesimally rigid. Roth and Whiteley (see the proof of [10, Theorem 6.3]) prove that abstract (generalized) Grünbaum polygons are robust (and hence also strong) and stable.

There exist tensegrity polygons without strict, or even non-trivial proper stress. It is not hard to construct examples in the case when the underlying abstract tensegrity polygon has a separating vertex pair (see Sect. 5). Other examples include (specific convex realizations of) the abstract tensegrity polygons of Fig. 4, see also [10, Sect. 6].

Roth and Whiteley [10, p. 441] note that "little seems to be known about what distinguishes cabling schemes which give infinitesimally rigid tensegrity polygons ${ }^{1}$ for all convex realizations from those that do not". Connelly [5] asked (see also [6, Sect. 10.2]) whether there is a good combinatorial characterization of the family of robust abstract tensegrity polygons $T=(V ; C, S)$, at least in the special case when $|S|=|V|-2$. Our main result is a characterization of those tensegrity polygons with $|V|-2$ struts for which these properties (infinitesimal rigidity, the existence of a non-zero or a strict proper stress) depend only on $T$ and are independent of the choice of $p$. We note that for all tensegrity polygons, with any number of struts, the concepts of strong, robust, and stable are all the same, see Sect. 1.2.

\subsection{Basic definitions and the main theorem}

Let $T=(V ; C, S)$ be a cable-strut tensegrity graph. We use $d_{S}(v)$ to denote the number of struts incident with a vertex $v \in V$ and call this number the strut-degree of $v$. The tensegrity graph $(V ; S)$ on the same vertex set but containing only the struts of $T$ is the strut graph of $T$. A connected component of the strut graph is a strut component.

Let $T=(V ; C, S)$ be an abstract tensegrity polygon with boundary cycle $H$. $H$ defines a cyclic ordering of $V$. An interval $I$ in $T$ is a maximal set of vertices satisfying that (i) each vertex in $I$ has strut-degree at least two, and (ii) the vertices

\footnotetext{
1 In their polygons the boundary cycle consists of struts, and the diagonals are cables. However, interchanging the cables and struts of a tensegrity framework preserves infinitesimal rigidity and also the existence of a strict proper stress.
} 
in $I$ are consecutive, i.e. they induce a subpath of $H$. A leaf is a strut $u v \in S$ with $d_{S}(v)=1$. Two struts $u_{1} v_{1}, u_{2} v_{2}$ cross each other if they are crossing diagonals of the boundary cycle $H$. (Incident struts do not cross.) Suppose that two leaves incident with the same interval $I$ cross. Then we say the crossing pair of leaves is admissible with respect to $I$ if their end-vertices on $I$ are consecutive, i.e. they are connected by a cable. For example, the third graph of Fig. 3 contains four pairs of crossing leaves and they are all admissible.

We say that $T$ satisfies the unique interval property if:

(a) $T$ has exactly one interval $I$,

(b) either $|I|=1$ and $T$ is an abstract Grünbaum polygon or $|I| \geq 2$ and one end-vertex of each leaf belongs to $I$,

(c) each pair of vertices of $I$ whose distance is two along $H$ is connected by a strut and there are no other struts induced by $I$,

(d) each pair of crossing leaves is admissible with respect to $I$.

It is easy to check, from the algorithmic point of view, whether a given abstract tensegrity polygon satisfies the unique interval property.

Note that (c) is equivalent to saying that the restriction of the strut graph to the vertices of $I$ is the union of two disjoint paths, whose vertices alternate on the boundary cycle. It is easy to check that if $T$ satisfies the unique interval property then its underlying graph is 3-connected and it has at most $|V|-2$ struts. Furthermore, if $|S|=|V|-2$ holds then each vertex in $V-I$ is incident with a leaf. For example, the Cauchy and the generalized Grünbaum polygons all satisfy the unique interval property. The polygons in Fig. 4 do not. The abstract tensegrity polygon on four vertices (Fig. 1) has no interval.

Our main result is as follows.

Theorem 1.2 Let $T=(V ; C, S)$ be an abstract tensegrity polygon with $|V| \geq 5$ and $|S|=|V|-2$. Then the following are equivalent:

(i) $T$ is strong,

(ii) $T$ is robust,

(iii) $T$ is stable,

(iv) $T$ satisfies the unique interval property.

In Sect. 2 we verify some basic properties of (abstract) tensegrity polygons. In Sect. 3 we consider strong polygons and prove that (i) implies (iv) in Theorem 1.2. We show that (iv) implies (ii) and (iii) in Sect. 4. Since (iii) implies (ii) and (ii) implies (i), this will complete the proof of Theorem 1.2. We make some concluding remarks in Sect. 5.

\subsection{Previous Work}

In the rest of this section we provide a brief summary of some previous results on tensegrity polygons and their applications as well as some remarks on potential applications of robust polygons and our characterization.

We start with the fact that, as we remarked earlier, strong, robust, and stable are the same for tensegrity polygons. Stable implies robust by Theorem 1.1, while robust 
implies strong by definition. The following argument, suggested by Bob Connelly, shows that strong implies stable: in [2, Corollary 1] he proved that if a tensegrity polygon $(T, p)$ has a non-trivial proper stress then it is universally rigid (or uniquely embedded), which means that every other realization of $T$, in any dimension $d \geq 2$, in which the cables are not longer and the struts are not shorter than the corresponding members in $(T, p)$, is congruent to $(T, p)$, i.e. it can be obtained from $(T, p)$ by restricting a rigid motion of $\mathbb{R}^{d}$ to the vertices of $(T, p)$. Now suppose that $q$ is a non-trivial infinitesimal motion of a convex realization $(T, p)$ of a strong abstract tensegrity polygon $T$. Then, by using the Averaging Theorem [11, Theorem 60.1.32], it follows that $(T, p+q)$ and $(T, p-q)$ are equivalent, but not congruent convex realizations of $T$, assuming that $q$ is properly scaled. But since $T$ is strong, these realizations also have a non-zero proper stress, and hence they are universally rigid, a contradiction. Thus strong implies stable, as claimed.

In a different paper Connelly [4] proved that Cauchy polygons are infinitesimally rigid and used this fact in the proof of his main result, saying that any triangulated convex surface is rigid. As noted earlier, Roth and Whiteley [10] analysed even larger families of tensegrity polygons and made several fundamental observations. Connelly and Whiteley [7] introduced the concepts of prestress stability and second-order rigidity for tensegrity frameworks and showed that infinitesimal rigidity implies prestress stability, which implies second-order rigidity, which implies rigidity. They also verified the reverse implications for certain tensegrity polygons. Additional rigidity properties of (not necessarily convex) realizations of the underlying graphs of Cauchy and Grünbaum polygons are mentioned in [8]. For further open questions and conjectures the reader is referred to [6].

It is well-known that (infinitesimal) rigidity of tensegrity frameworks is not a generic property, unlike in the special case of bar frameworks. In fact it is an open problem, even in two dimensions, to characterize those tensegrity graphs which have infinitesimally rigid realizations (and to find such a realization, if there is one). See [9] for a survey of related results and "form-finding" methods. This motivates the search for families of tensegrity frameworks for which certain rigidity properties are generic, at least within some reasonable class of realizations. Properties of this kind, such as robustness, might be useful in these more general problems, too.

\section{Preliminaries}

In this section we prove some preliminary results which are valid for abstract tensegrity polygons with an arbitrary number of struts.

First we recall a simple and useful technical lemma which can be used to analyse the signs of the stress coefficients at a vertex.

Lemma 2.1 [10, Lemma 6.2] Suppose $p_{j} \in \mathbb{R}^{2}-\{\mathbf{0}\}$ and $\omega_{j} \in \mathbb{R}-\{0\}$ for $1 \leq j \leq n$. If $\sum_{j=1}^{n} \omega_{j} p_{j}=\mathbf{0}$ then there does not exist a line through the origin such that $\left\{p_{j}: \omega_{j}>0\right\}$ is contained in one open half space determined by the line and $\left\{p_{j}: \omega_{j}<0\right\}$ is contained in the other open half space.

Let $(T, p)$ be a tensegrity polygon and let $\omega$ be a non-trivial proper stress on $(T, p)$. Consider a vertex $v_{i}$ which is incident with a member $v_{i} v_{j}$ for which $\omega_{i j}$ is non-zero. 
Convexity and Lemma 2.1 imply that the stress in both cables incident with $v_{i}$ must be positive. This in turn implies that $v_{i}$ must be incident with at least one strut (in which the stress is negative). By applying this argument around the boundary cycle we obtain:

Lemma 2.2 Let $(T, p)$ be a tensegrity polygon and let $\omega$ be a non-trivial proper stress on $(T, p)$. Then $\omega$ is non-zero on every cable of $T$ and every vertex is incident with a strut.

We shall frequently rely on the next key lemma in our proofs.

Lemma 2.3 Let $T$ be a strong abstract tensegrity polygon and suppose that $v v_{1}$ and $v v_{2}$ are incident cables of $T$ such that there is no strut between $v_{1}$ and $v_{2}$. Then the abstract tensegrity polygon $T^{\prime}$ obtained from $T$ by deleting $v$ and adding a new cable $v_{1} v_{2}$ is also strong.

Proof First note that since there is no strut between $v_{1}$ and $v_{2}, T^{\prime}$ is indeed an abstract tensegrity polygon. Let $\left(T^{\prime}, p\right)$ be a convex realization of $T^{\prime}$. We shall prove that there is a non-trivial stress on $\left(T^{\prime}, p\right)$. Consider a sequence $\left(T, p_{k}\right), k \geq 1$, of convex realizations of $T$ satisfying that $p_{k}(u)=p(u)$ for all $u \in V-\{v\}$ and $k \geq 1$, and that $p_{k}(v)$ converges to the midpoint of the segment $\left[p\left(v_{1}\right), p\left(v_{2}\right)\right]$. Since $T$ is strong, $\left(T, p_{k}\right)$ has a non-trivial proper stress $\omega_{k}$ for all $k \geq 1$. By multiplying some of the stresses, if necessary, we may suppose that $\max \left\{\left|\omega_{k}(e)\right|: e \in C \cup S\right\}=1$ holds for all $k \geq 1$. Since all the stress values are between -1 and 1 , we can find a subsequence $\omega_{l}, l \geq 1$, for which $\omega_{l}(e)$ converges to some number $\omega_{\text {lim }}(e)$ for all members $e$ of $T$. It is easy to see that $\omega_{\text {lim }}$ is a proper stress on the limit framework $\left(T, p_{\text {lim }}\right)$.

Now we define a proper stress $\omega$ on $\left(T^{\prime}, p\right)$. For all members $e$ of $T^{\prime}$, except for $v_{1} v_{2}$, we let $\omega(e)=\omega_{\text {lim }}(e)$, while we define $\omega\left(v_{1} v_{2}\right)=\frac{1}{2} \omega_{\text {lim }}\left(v v_{1}\right)$. Since $p_{\text {lim }}\left(v_{1}\right), p_{\text {lim }}\left(v_{2}\right)$ and $p_{\text {lim }}(v)$ are collinear, we must have $\omega_{\text {lim }}\left(v v_{x}\right)=0$ for all struts $v v_{x}$ incident with $v$. Moreover, $\omega_{\text {lim }}\left(v v_{1}\right)=\omega_{\text {lim }}\left(v v_{2}\right)$. These facts imply that $\omega$ is indeed a proper stress on $\left(T^{\prime}, p\right)$.

We can also observe that $\omega$ is non-trivial, since the absolute value of the stress on at least one member is equal to 1 for every $\omega_{k}$, and hence also for every $\omega_{l}$.

We say that a vertex $v$ is strut-covered if there is a strut between the cable-neighbours of $v$. The operation of Lemma 2.3, which deletes a vertex $v$, which is not strut-covered, and adds a new cable connecting its cable-neighbours, is called a reduction at $v$.

Lemma 2.4 Let $T$ be an abstract tensegrity polygon with at least three strut components. Then $T$ is not strong.

Proof For a contradiction suppose that $T$ is strong. By applying reductions as long as possible we may assume, by Lemma 2.3, that for each vertex $v$ we have that either $v$ is strut-covered (and hence no reduction is possible at $v$ ) or a reduction at $v$ decreases the number of strut components to two.

Suppose that we have a sequence of at least two vertices along the boundary cycle whose vertices belong to the same strut component. Consider a maximal sequence of this type. It is easy to check that the first vertex $v$ of this interval cannot be strutcovered and a reduction at $v$ does not decrease the number of strut components. This contradicts our previous assumption. 
Hence any two consecutive vertices along the boundary cycle belong to different strut components and therefore we must have three consecutive vertices belonging to different strut components. The middle vertex $v$ of this consecutive triple cannot be strut-covered, so, by our assumption, a reduction at $v$ should decrease the number of strut components. However, this number can decrease only if $v$ is a singleton component in the strut graph of $T$, which contradicts the fact that $T$ is strong by Lemma 2.2. This completes the proof of the lemma.

Lemma 2.4 implies that every strong (and hence every robust) abstract tensegrity polygon on vertex set $V$ has at least $|V|-2$ struts.

Lemma 2.5 Let $T$ be a strong abstract tensegrity polygon with two strut components. Suppose that $v$ is a cut-vertex in one of the strut components. Then $v$ is strut-covered.

Proof Suppose $v$ is not strut-covered and perform a reduction at $v$. By Lemma 2.3 the resulting abstract tensegrity polygon $T^{\prime}$ is strong. On the other hand, since $v$ is a cut-vertex in a strut component, the number of strut components of $T^{\prime}$ is at least three. This implies that $T^{\prime}$ is not strong by Lemma 2.4, a contradiction.

\section{Strong Polygons with $|V|-2$ Struts}

In this section we first summarize some useful properties of strong abstract tensegrity polygons with $|V|-2$ struts and then prove that such polygons satisfy the unique interval property.

Lemma 3.1 Let $T=(V ; C, S)$ be a strong abstract tensegrity polygon with $|S|=$ $|V|-2$. Then

(i) the strut graph of $T$ is a forest with two components,

(ii) if $|V| \geq 5$ then $T$ has at least one interval,

(iii) if $v \in V$ belongs to some interval then $v$ is strut-covered,

(iv) for an interval I of $T$ we have $|I| \leq|V|-4$,

(v) the four vertices on the boundary cycle next and second-next to I have strutdegree one, and the second-next vertices are not strut-covered,

(vi) if $|V| \geq 6$ and $I=\{w\}$ is a singleton interval then $d_{S}(w) \geq 3$.

Proof (i) Lemma 2.4 implies that $T$ has at most two strut components. Since $|S|=$ $|V|-2$ this is possible only if the strut graph is a forest with two components.

(ii) If $T$ has no interval then each vertex $v$ has $d_{C}(v)=2$ and $d_{S}(v)=1$, which implies that the underlying graph is three-regular. With $2|V|-2$ members this can happen only if $|V|=4$.

(iii) Since the strut graph is a forest, each vertex $v$ with $d_{S}(v) \geq 2$ is a cut-vertex in its strut component. Thus Lemma 2.5 implies that all vertices of an interval are strut-covered. 
(iv) Consider an interval $I$. Since $d_{S}(v) \geq 1$ for all $v \in V$ by Lemma 2.2, we have

$$
2|S|=2|V|-4=\sum_{v \in V} d_{S}(v) \geq 2|I|+|V|-|I|,
$$

and hence $|I| \leq|V|-4$ follows.

(v) Let $a, b$ denote the vertices next and second-next to $I$ on the boundary cycle at some end of $I$. By the maximality of $I$ we have $d_{S}(a)=1$. Since each vertex of $I$ is strut-covered, the strut incident with $a$ is the one that covers the vertex of $I$ next to $a$. Thus $b$ cannot be strut-covered, since this strut covering $b$ would also be incident with $a$. This implies $d_{S}(b)=1$ by (iii).

(vi) Suppose that $d_{S}(w)=2$ for some vertex $w$ which forms an interval by itself. Let $a$ and $a^{\prime}$ be the cable-neighbours of $w$ and let $b, b^{\prime}$ be the vertices second-next to $w$ on the boundary cycle (with $b$ the other cable-neighbour of $a$, say). Since $w$ is strut-covered, $a a^{\prime} \in S$ holds. By (v) $b$ is not strut-covered. If $a$ and $a^{\prime}$ are both strut-covered then apply a reduction at $b$, which leads to a strong tensegrity polygon by Lemma 2.3. But this reduction creates two strut components of size two (consisting of the struts $a a^{\prime}$ and $w b^{\prime}$, respectively), and hence at least three strut-components in total, when $|V| \geq 6$. This contradicts Lemma 2.4. So we may suppose that $a$ is not strut-covered. Now a reduction at $a$ makes the strut-degree of $a^{\prime}$ zero, a contradiction by Lemmas 2.2 and 2.3.

Lemma 3.2 Let $T=(V ; C, S)$ be a strong abstract tensegrity polygon with boundary cycle $H$ and with $|S|=|V|-2$ Suppose that $T$ has exactly one interval I. Then:

(i) if $|I|=1$ then $T$ is an abstract Grünbaum polygon,

(ii) if $|I| \geq 2$ then one end-vertex of each leaf belongs to $I$,

(iii) each pair of vertices of I whose distance is two along $H$ is connected by a strut and there are no other struts induced by $I$.

Proof (i) If there is only one vertex $w \in V$ with strut-degree at least two then the strut graph must be the union of a star and an edge by Lemma 3.1(i). Since $w$ is strut-covered by Lemma 3.1(iii), this implies that $T$ is an abstract Grünbaum polygon.

(ii) Let $x y$ be a leaf with $d_{S}(x)=1$. If $x$ is a vertex next to $I$ then the leaf incident with it must be the strut that covers the last vertex of $I$ by Lemma 3.1(iii). Since $|I| \geq 2$, this gives $y \in I$. Now consider the case when $x$ is not next to $I$ and, for a contradiction, suppose that $y \notin I$. Then $d_{S}(y)=1$ and hence we may also assume that $y$ is not next to $I$. Observe that if $x$ is strut-covered, say, then the strut covering $x$ is also a leaf with both end-vertices having strut-degree one. This means the strut graph has at least three components, a contradiction by Lemma 2.4. Thus we may perform a reduction at $x$, which makes the strut-degree of $y$ zero. This contradicts Lemmas 2.2 and 2.3.

(iii) By Lemma 3.1(iii) each vertex of $I$ is strut-covered. Thus each pair of vertices of $I$ whose distance is two along $H$ is connected by a strut. This implies that the strut subgraph on $I$ has at most two components, since it contains two disjoint paths, whose union is $I$ and whose vertices alternate along the boundary cycle. If 
there is an additional strut within this subgraph then either we have a strut cycle (which is impossible by Lemma 3.1(i)) or all vertices in this subgraph belong to the same strut component. This would mean, by (ii), that the strut graph is connected, a contradiction.

Lemma 3.3 Let $T$ be a strong abstract tensegrity polygon with $|S|=|V|-2$ and $|V| \geq 5$. Then $T$ has exactly one interval $I$.

Proof Lemma 3.1(ii) implies that $T$ has at least one interval. Suppose, for a contradiction, that $T$ has at least two intervals. Let us perform reductions at vertices of strut-degree one as long as possible preserving the existence of at least two intervals. By Lemma 2.3 the resulting abstract tensegrity polygon $T^{\prime}$ is also strong. We may assume that $T^{\prime}$ has at least six vertices, since otherwise it cannot have two vertices of strut-degree two. Consider $T^{\prime}$ and choose an interval $I$ of $T^{\prime}$. Let $a, b$ be the next and second-next vertices to $I$, respectively, at one of its ends. By Lemma 3.1(v) we have $d_{S}(b)=1$ and $b$ is not strut-covered. This means that we may apply a reduction at $b$ - however, by the choice of $T^{\prime}$, a reduction at $b$ would lead to a polygon with one interval. Let $b x$ be the strut incident with $b$.

Since the intervals in $T^{\prime}$ are separated from each other by at least two vertices along the boundary cycle, by Lemma 3.1(iv), the reduction at $b$, which may decrease the strut-degree of $x$ only, can decrease the number of intervals only if there is an interval consisting only of vertex $x$, and $d_{S}(x)=2$. But Lemma 3.1(vi) shows that the vertex of a singleton interval has strut degree at least three, a contradiction.

Lemma 3.4 Let $T$ be a strong abstract tensegrity polygon with $|S|=|V|-2$ and $|V| \geq 5$ and let $I$ be an interval of $T$. Then each pair of crossing leaves is admissible with respect to $I$.

Proof By Lemma $3.3 I$ is the only interval in $T$. Suppose, for a contradiction, that there is a pair of crossing leaves which is non-admissible with respect to $I$. Let us perform reductions at vertices of strut-degree one as long as possible preserving the existence of a non-admissible crossing pair with respect to the unique interval of the polygon. By Lemma 2.3 the resulting abstract tensegrity polygon $T^{\prime}$ is also strong. Consider $T^{\prime}$ and its interval $I$. We can use Lemma 3.2 and the fact that there are no crossing leaves with respect to the interval of a Grünbaum polygon to deduce that $|I| \geq 2$ and all leaves of $T^{\prime}$ are incident with $I$. In fact, we must have $|I| \geq 3$, since otherwise all crossings must be admissible.

Let $a, b$ be the next and second-next vertices to $I$, respectively, at one of its ends. By Lemma 3.1(v) we have $d_{S}(b)=1$ and $b$ is not strut-covered. Hence we may apply a reduction at $b$. However, by the choice of $T^{\prime}$, a reduction at $b$ would lead to a polygon without non-admissible pairs of crossing leaves. Let $b x$ be the strut incident with $b$. We have $x \in I$. By Lemma 3.2(iii) it follows that the reduction at $b$ cannot decrease the strut-degree of the inner vertices of $I$ to one. Furthermore, the strut-degree of an end-vertex $v$ of $I$ is decreased to one (and hence the interval becomes shorter) if and only if $d_{S}(v)=2$ and $x=v$ hold. In this case $v$ is eliminated from $I$, i.e. the interval of the reduced polygon is $I-\{v\}$.

Suppose first that $b x$ is not part of a non-admissible pair of crossing leaves in $T^{\prime}$. Then the reduction at $b$ preserves the existence of a non-admissible crossing pair. This 
follows by observing that if $I$ remains the same then the set of non-admissible pairs of crossing leaves cannot change. If $I$ gets shorter then we must have $d_{S}(v)=2$ and $x=v$ for an end-vertex $v$ of $I$. However, no strut incident with $v$ can be part of a non-admissible crossing pair (as one of them is $b v$ and the other is the strut that covers the vertex next to $v$ in $I$ ), and again the set of non-admissible pairs of crossing leaves remains the same. This contradicts the choice of $T^{\prime}$.

So it remains to consider the case when $b x$ is part of a non-admissible pair of crossing leaves in $T^{\prime}$. Then $a$ cannot be strut covered and hence we may apply a reduction at $a$. By Lemma 2.3 the polygon obtained by the reduction at $a$ is strong, and hence it has exactly one interval by Lemma 3.3. Since the strut $a y$ incident with $a$ is the strut covering the last vertex of $I$, and $|I| \geq 3$, the vertex set of the interval does not change by the reduction. Since ay cannot be in a non-admissible pair of crossing leaves in $T^{\prime}$, this implies that non-admissible pairs remain non-admissible after the reduction at $a$. This contradicts the choice of $T^{\prime}$, which completes the proof.

The results of this section show that if an abstract tensegrity polygon $T$ is strong then it satisfies the unique interval property. Namely, Lemmas 3.3, 3.2, and 3.4 imply properties (a), (b) and (c), and (d), respectively.

\section{Robust Polygons with $|V|-2$ Struts}

In this section we show that if $T$ satisfies the unique interval property then $T$ is robust.

Lemma 4.1 Let $T=(V ; C, S)$ be an abstract tensegrity polygon with $|S|=|V|-2$ and $|V| \geq 6$. Suppose that $T$ satisfies the unique interval property and the interval $I$ of $T$ has at least two vertices. Let $v_{k-1}, v_{k}$ be the last two vertices of I at one of its ends and let $a, b$ be the next and second-next vertex to I on the boundary cycle. Then

(i) there is exactly one strut incident with $b$, which is either $v_{k} b$ or $v_{k-1} b$,

(ii) if $v_{k} b \in S$ then $b$ is not strut-covered and a reduction at $b$ preserves the unique interval property,

(iii) if $v_{k-1} b \in S$ then a is not strut-covered and a reduction at a preserves the unique interval property.

Proof (i) As we noted earlier, the unique interval property and $|S|=|V|-2$ implies that each vertex in $V-I$ is incident with exactly one strut. Since $v_{k}$ is the last vertex of $I$, at most one strut leads from $v$ to another vertex in $I$ and hence $v_{k}$ is incident with a leaf $v_{k} y$. Now either $b=y$ and hence $v_{k} b$ is a strut, or the strut incident with $b$ and $v_{k} y$ cross with respect to $I$. In the latter case, by the unique interval property, $v_{k-1} b$ must be a strut, for otherwise this crossing pair would be non-admissible with respect to $I$.

(ii) Suppose $v_{k} b \in S$. The unique interval property implies that $v_{k-1} a$ is a strut, hence $b$ cannot be strut-covered. Let $T^{\prime}$ be obtained from $T$ by a reduction at $b$. The strut-degrees do not change, except at $v_{k}$, where it is decreased by one. If its strut-degree in $T^{\prime}$ is at least two, the interval of $T^{\prime}$ is identical to $I$, in which case it is easy to see that properties (a)-(d) are all maintained. If the strut-degree of $v_{k}$ drops to one by the reduction, then the interval of $T^{\prime}$ is $I$ minus $v_{k}$. If the interval 
of $T^{\prime}$ is a single vertex, then the unique interval property of $T$ can be used to deduce that $T^{\prime}$ is an abstract Grünbaum polygon. If the interval of $T^{\prime}$ is of size at least two then again, it is easy to see that properties (a)-(d) are all maintained.

(iii) Suppose $v_{k-1} b \in S$. In this case $a$ cannot be strut-covered. The unique interval property implies that the strut incident with $a$ is $v_{k-1} a$. Let $T^{\prime}$ be obtained from $T$ by a reduction at $a$. If $|I| \geq 3$ then, by using the unique interval property, we have $v_{k-3} v_{k-1} \in S$. Since $v_{k-1} b \in S$, it follows that in this case the strutdegree of $v_{k-1}$ is at least two in $T^{\prime}$. Hence the interval of $T^{\prime}$ is identical to $I$, in which case it is easy to see that properties (a)-(d) are all maintained. If $|I|=2$ then either the strut degree of $v_{k-1}$ is at least two in $T^{\prime}$, in which case we are done as above, or we have $d_{S}\left(v_{k-1}\right)=2$ in $T$. In the latter case the unique interval property of $T$ implies that $T^{\prime}$ is a Grünbaum polygon. This completes the proof.

In the next proof we shall use the following operation. Suppose that we have two tensegrity frameworks $\left(T_{1}, p_{1}\right)$ and $\left(T_{2}, p_{2}\right)$, possibly with overlapping sets of vertices, and corresponding stresses $\omega_{1}$ and $\omega_{2}$. Then we can define a stress $\omega$ on the (underlying bar framework of the) union of the two frameworks by first extending each stress by zeros on the members of the other frameworks, and then taking their sum. We say we superimpose the stresses when we apply this operation (which was used already in [10]).

Theorem 4.2 Let $T$ be an abstract tensegrity polygon with $|S|=|V|-2$ and $|V| \geq 5$. Suppose that $T$ satisfies the unique interval property. Then $T$ is robust and stable.

Proof The proof is by induction on $|V|$. If $|V|=5$ then $T$ is an abstract Grünbaum polygon, which is known to be robust and stable. So suppose that $|V| \geq 6$ and consider a convex realization $(T, p)$ of $T$. We shall construct a strict proper stress on $(T, p)$ and show that it is infinitesimally rigid by superimposing (the strict proper stresses of) two smaller tensegrity polygons.

Since $T$ satisfies the unique interval property and Grünbaum polygons are robust and stable, we may suppose that $|I| \geq 2$. Let $v_{k-1}, v_{k}$ be the last two vertices of $I$ at one of its ends and let $a, b$ be the next and second-next vertex to $I$ on the boundary cycle. By Lemma 4.1(i) there is exactly one strut incident with $b$, which is either $v_{k} b$ or $v_{k-1} b$. We shall deal with these two cases separately.

Case $1 v_{k} b$ is a strut.

Create a smaller tensegrity polygon $\left(T^{\prime}, p^{\prime}\right)$ by deleting $a$, replacing the strut $v_{k} b$ by a cable and adding a new strut $v_{k-1} b$, without changing the vertex positions. Let $\left(K_{4}, p\right)$ be a tensegrity polygon on four vertices $p\left(v_{k-1}\right), p\left(v_{k}\right), p(a), p(b)$ with struts $v_{k-1} a$ and $v_{k} b$. Observe that the underlying abstract tensegrity polygon $T^{\prime}$ is isomorphic to the one obtained from $T$ by a reduction at $b$. Thus $T^{\prime}$ satisfies the unique interval property by Lemma 4.1. Hence, by induction, it is robust and stable. So is the small tensegrity polygon. Thus both $\left(T^{\prime}, p^{\prime}\right)$ and $\left(K_{4}, p\right)$ have a strict proper stress and are infinitesimally rigid. By scaling the stresses, if necessary, we may assume that the sum of the stresses on the strut $v_{k-1} b$ and on the cable $v_{k-1} b$ in the corresponding frameworks is zero. Now consider the superimposed stress $\omega$ on the union of the two frameworks, see Fig. 5. 


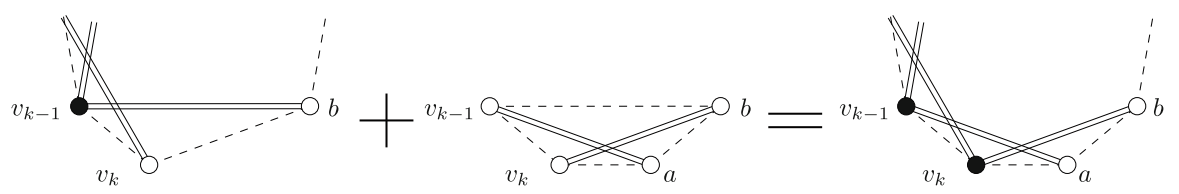

Fig. 5 Superimposing the (stresses of the) two polygons in Case 1

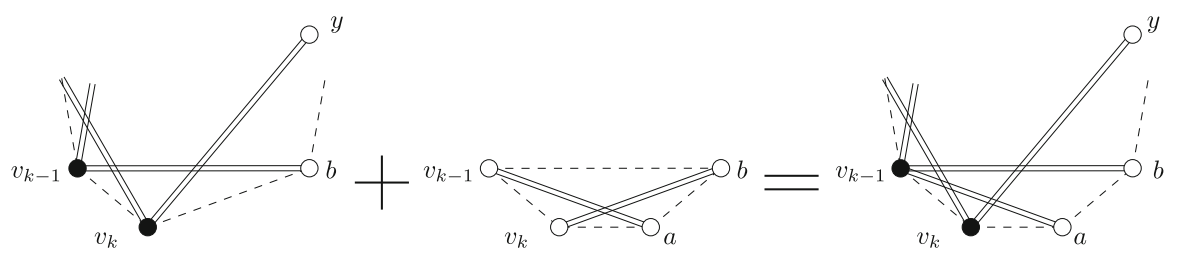

Fig. 6 Superimposing the (stresses of the) two polygons in Case 2

By our assumption $\omega$ is non-zero only on the members of $(T, p)$ and its sign is consistent with the labeling of $T$ except, possibly, on the member $v_{k} b$, where we take the sum of two numbers of opposite signs. However, since $\omega$ is positive on the members of the boundary cycle of $T$ and $v_{k} b$ is the only other member incident with $b$, it follows that the $\omega$ is negative on $v_{k} b$. Therefore $\omega$ is a strict proper stress on $(T, p)$, as required. Furthermore, since $\left(T^{\prime}, p^{\prime}\right)$ and $\left(K_{4}, p\right)$ are both infinitesimally rigid, we can use [10, Theorem 6.1$]$ to deduce that $(\bar{T}, p)$ is infinitesimally rigid. This fact, the existence of $\omega$, and Theorem 1.1 imply that $(T, p)$ is infinitesimally rigid.

Case $2 v_{k-1} b$ is a strut.

By Lemma 4.1(iii) $a$ is not strut-covered. Create a smaller tensegrity polygon $\left(T^{\prime}, p^{\prime}\right)$ by reducing $T$ at $a$, without changing the vertex positions. Let $\left(K_{4}, p\right)$ be the polygon on four vertices $p\left(v_{k-1}\right), p\left(v_{k}\right), p(a), p(b)$ with struts $v_{k-1} a$ and $v_{k} b$. By Lemma 4.1(iii) $T^{\prime}$ also satisfies the unique interval property. Hence, by induction, it is robust and stable. So is the small tensegrity polygon. Thus both $\left(T^{\prime}, p^{\prime}\right)$ and $\left(K_{4}, p\right)$ have a strict proper stress and are infinitesimally rigid. By scaling the stresses, if necessary, we may assume that the sum of the stresses on the strut $v_{k} b$ and on the cable $v_{k} b$ in the corresponding frameworks is zero. Now consider the superimposed stress $\omega$ on the union of the two frameworks, see Fig. 6.

By our assumption $\omega$ is non-zero only on the members of $(T, p)$ and its sign is consistent with the labeling of $T$ except, possibly, on the member $v_{k-1} b$, where we take the sum of two numbers of opposite signs. However, since $\omega$ is positive on the members of the boundary cycle of $T$ and $v_{k-1} b$ is the only other member incident with $b$, it follows that $\omega$ is negative on $v_{k-1} b$. Therefore $\omega$ is a strict proper stress on $(T, p)$, as required. Furthermore, since $\left(T^{\prime}, p^{\prime}\right)$ and $\left(K_{4}, p\right)$ are both infinitesimally rigid, we can use $[10$, Theorem 6.1$]$ to deduce that $(\bar{T}, p)$ is infinitesimally rigid. This fact, the existence of $\omega$, and Theorem 1.1 imply that $(T, p)$ is infinitesimally rigid. This completes the proof.

We are now ready to prove the main result. 
Proof (of Theorem 1.2) Let $T=(V ; C, S)$ be an abstract tensegrity polygon with $|V| \geq 5$ and $|S|=|V|-2$. First suppose that $T$ is strong. Then $T$ satisfies the unique interval property by Lemmas 3.2, 3.3, and 3.4. Thus (i) implies (iv).

Next suppose that $T$ satisfies the unique interval property. Then $T$ is robust and stable by Theorem 4.2. This shows that (iv) implies (iii) and (ii). Finally, we can see that (iii) implies (ii) by Theorem 1.1 and (ii) implies (i) by definition. This completes the proof.

\section{Concluding Remarks}

Theorem 1.2 can be used to deduce a similar combinatorial characterization concerning reversed (abstract) tensegrity polygons, which are obtained by interchanging the cables and struts in an (abstract) tensegrity polygon, and rigidity. Connelly and Whiteley [7, Proposition 6.2.1] proved that such a reversed tensegrity polygon is rigid if and only if it is infinitesimally rigid. (They consider the case when the members on the boundary cycle are bars but it is not hard to see that their result is valid in the struts and cables version, too.) Since interchanging the cables and struts preserves infinitesimal rigidity, we obtain the following result.

Theorem 5.1 Let $T=(V ; S, C)$ be an abstract reversed tensegrity polygon with $|V| \geq 5$ and $|C|=|V|-2$. Then every convex realization $(T, p)$ is rigid if and only if $T$ satisfies the reversed unique interval property.

Let $T=(V ; C, S)$ be a robust (or stable) abstract tensegrity polygon with $|V|-2$ struts. The proof of Theorem 4.2 shows that $T$ can be reduced to the abstract tensegrity polygon on four vertices by reductions. Therefore the underlying undirected graph $G$ of $T$ can be obtained from $K_{4}$ by repeated applications of the (unlabeled) inverse operation of reduction. This operation, which is called 1-extension (or edge-splitting), builds up a 3-connected, generically redundantly rigid, and generically globally rigid graph from $K_{4}$, see $[1,3]$. Thus $G$ satisfies each of these properties.

Let $T=(V ; C, S)$ be an abstract tensegrity polygon which is not strong. It may be interesting to find a method for verifying this by constructing a tensegrity polygon $(T, p)$ which has no non-trivial proper stress. If $T$ is not 3-connected then this can be done as follows. Let $\left\{v_{i}, v_{j}\right\}$ be a separating vertex pair in $T$ with $i<j$. Build a convex realization $(T, p)$ of $T$ in such a way that all struts leaving the vertex set $R=\left\{v_{i+1}, v_{i+2}, \ldots, v_{j}\right\}$ go up and left, while both cables leaving $R$ go up and right. See Fig. 7, where $R$ is the set below the line. Consider a proper stress $\omega$ on $(T, p)$. By taking the sum of the equilibrium equations (1) over the vertices of $R$ we can deduce that the sum of the vectors (scaled with $\omega$ ) corresponding to the members leaving $R$ must also be the zero vector. However, the directions chosen for these members imply that the horizontal component in each of these vectors is negative, and hence the stress in each of these members must be zero. In particular, the stress is zero in the cables leaving $R$. Thus $\omega$ is the zero stress by Lemma 2.2 .

A straightforward open problem is to extend the combinatorial characterization of this paper to the case when we may have more struts in our polygons. It is clear from Theorem 1.2 that if an abstract tensegrity polygon $T=(V ; C, S)$ contains a subgraph 
Fig. 7 A tensegrity polygon without non-trivial proper stresses. The separating vertex pair is $\left\{v_{i}, v_{j}\right\}$

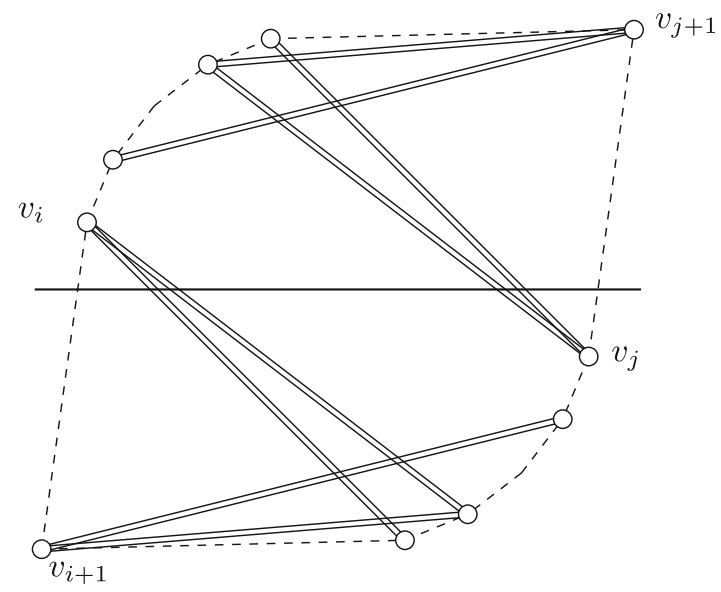

with $|V|-2$ struts which satisfies the unique interval property then $T$ is robust. It is not clear how to test whether there is such a subgraph. Furthermore, even if we could test the existence of such subgraphs, it would not solve the problem. It is due to the fact that there exist robust abstract tensegrity polygons without such sparse robust subgraphs: consider, for example, a polygon on seven vertices in which each long diagonal is a strut (so the strut graph is also a cycle of length seven).

Acknowledgments We thank Viktória Kaszanitzky and Csaba Király for carefully reading our manuscript and for several useful comments. This work was supported by the MTA-ELTE Egerváry Research Group on Combinatorial Optimization, the Hungarian Scientific Research Fund Grant No. K81472, and the National Development Agency of Hungary, Grant No. CK 80124, based on a source from the Research and Technology Innovation Fund.

\section{References}

1. Berg, A.R., Jordán, T.: A proof of Connelly's conjecture on 3-connected circuits of the rigidity matroid. J. Comb. Theory Ser. B 88(1), 77-97 (2003)

2. Connelly, R.: Rigidity and energy. Invent. Math. 66, 11-33 (1982)

3. Connelly, R.: Generic global rigidity. Discrete Comput. Geom. 33, 549-563 (2005)

4. Connelly, R.: The rigidity of certain cabled frameworks and the second-order rigidity of arbitrarily triangulated convex surfaces. Adv. Math. 37(3), 272-299 (1980)

5. Connelly, R.: Recent progress in rigidity theory, workshop at BIRS. Banff, Canada, July (2008)

6. Connelly, R.: Questions, conjectures and remarks on globally rigid tensegrities, manuscript, November (2009)

7. Connelly, R., Whiteley, W.: Second-order rigidity and prestress stability for tensegrity frameworks. SIAM J. Discret. Math. 9(3), 453-491 (1996)

8. Jordán, T., Szabadka, Z.: Operations preserving the global rigidity of graphs and frameworks in the plane. Comput. Geom. 42(6-7), 511-521 (2009)

9. Juan, S.H., Mirats Tur, J.M.: Tensegrity frameworks: Static analysis review. Mech. Mach. Theory 43, 859-881 (2008)

10. Roth, B., Whiteley, W.: Tensegrity frameworks. Trans. Am. Math. Soc. 265(2), 419-446 (1981)

11. Whiteley, W.: Rigidity and scene analysis. In: Goodman, J.E., O'Rourke, J. (eds.) Handbook of Discrete and Computational Geometry, 2nd edn, pp. 1327-1354. CRC Press, Boca Raton (2004) 